\title{
Progressive nature of myocardial fibrosis in pediatric hypertrophic cardiomyopathy: from mutation carrier to myocardial fibrosis
}

\author{
Hong-Mi Choi ${ }^{1}$, Hyung-Kwan Kim $^{2}$ \\ ${ }^{1}$ Division of Cardiology, Hallym Sacred Heart Hospital, Hallym University College of Medicine, Anyang, Republic of Korea; ${ }^{2}$ Division of \\ Cardiology, Cardiovascular Center, Department of Internal Medicine, Seoul National University College of Medicine, Seoul, Republic of Korea \\ Correspondence to: Hyung-Kwan Kim, MD, PhD, Professor. Director of Cardiac Diagnostic Test Unit, Cardiovascular Center, Division of Cardiology, \\ Department of Internal Medicine, Seoul National University College of Medicine, 101 Daehak-ro, Jongno-gu, Seoul 03080, Korea. Email: \\ cardiman73@gmail.com or hkkim73@snu.ac.kr. \\ Provenance: This is an invited Editorial commissioned by Section Editor Kaiping Zhang, $\mathrm{PhD}$ (AME College, AME Group, China). \\ Comment on: Axelsson Raja A, Farhad H, Valente AM, et al. Prevalence and Progression of Late Gadolinium Enhancement in Children and \\ Adolescents with Hypertrophic Cardiomyopathy. Circulation 2018;138:782-92.
}

Submitted Sep 26, 2018. Accepted for publication Sep 30, 2018.

doi: 10.21037/atm.2018.10.07

View this article at: http://dx.doi.org/10.21037/atm.2018.10.07

Hypertrophic cardiomyopathy (HCM) is the most common genetic cardiomyopathy found in adults (1). Due to a wide variety of genetic defects and penetration, phenotypic manifestations or symptoms rise to the surface at a different time point during the lifetime (2). Early studies in adult HCM population focused on clinical manifestation and prognosis of patients with overt left ventricular hypertrophy (LVH). However, by virtue of easy accessibility to gene sequencing techniques and widely available family screening tool in HCM patients, researchers became interested in the genetically affected family members without overt LVH (3). From the perspective of a continuum of genetic disease, studies in the pediatric HCM patients and individuals carrying HCM-causing mutation without overt LVH (so-called preclinical or nonhypertrophic HCM) are of great importance to understand the pathophysiology and dynamic nature of HCM. Despite the absence of $\mathrm{LVH}$, abnormal test results such as ST-segment depression, decreased myocardial tissue velocity and the elevated NT-proBNP level were demonstrated in the preclinical stage of HCM population (4). In addition, pediatric HCM patients showing apparent LVH in the early childhood showed dismal outcome early after the diagnosis (5). Thus, the clinical features of pediatric HCM seem to be much more dynamic and diverse than those of adult HCM patients.

Cardiovascular magnetic resonance (CMR) is an emerging diagnostic tool for accurate differential diagnosis and risk stratification in a wide variety of myocardial diseases. Thanks to its unique ability of tissue characterization, especially detection and quantification of the myocardial scar using late gadolinium enhancement (LGE) (6), CMR has been adopted as a main evaluation tool in the assessment of HCM. Though not established as a sole risk factor, LGE was suggested to predict sudden cardiac death risk in adult HCM patients $(7,8)$. Due to a lack of the robust, single risk stratification strategy for sudden cardiac death, LGE has emerged as a highly possible prognosticator (9). Recently, in low-/intermediate-risk adult HCM patients (based on ESC sudden cardiac death criteria) with preserved left ventricular ejection fraction (10), LGE extent was significantly associated with prognosis, providing incremental prognostic utility (11). In a recent issue of Circulation, Axelsson Raja et al. reported CMR features of pediatric overt HCM and preclinical HCM population with a special focus on LGE (12). The authors addressed that LGE on CMR was detected in not a few pediatric HCM patients (46\%), and the presence of which was also associated with symptoms, adverse outcomes and ventricular tachycardia in pediatric HCM patients (13-15). Although the association between LGE and adverse outcome was not statistically significant in this study, the follow-up of 3.4 years might not be enough to see the link between LGE and adverse events considering that the study population is 
children. Furthermore, LGE did not exist in the preclinical HCM subjects in this study (12). This finding is consistent with the previous report of $40 \mathrm{HCM}$-causing mutation carriers (16). The notion that LGE, irreversible myocardial fibrosis, was not detected without overt LVH is consistent with our belief that a disease process may be continuous from simple genetic abnormality, the overt hypertrophic stage with myocardial fibrosis, to the final burn-out stage. Besides, maximal left ventricular wall thickness was the only independent predictor of LGE and its extent in overt HCM group (12), suggesting that myocardial fibrosis develops in the advanced stage of disease following LVH. They noted that 3 of 4 preclinical HCM patients (75\%) who underwent follow-up CMR scans showed progression to the overt HCM, a very high probability of progression (12). However, caution should be taken in the interpretation of reported progression rate of mutation carriers because not all preclinical HCM patients underwent CMR in this retrospective study. Notwithstanding, we need to keep in mind that disease progression does occur, requiring longitudinal follow-up. One more thing that we need to remember is that not only LVH but also myocardial fibrosis is a dynamic phenomenon $(17,18)$, and thus the time when CMR is performed can be a determinant of the presence or extent of LGE. This is why the incidence and extent of LGE should be interpreted with care in crosssectional studies.

Genetic factor or type of mutation is another component determining the onset of $\mathrm{LVH}$ and outcomes in individual adult HCM patients (19). Because sarcomeric proteins produced by each mutation have a distinct role in the myocardial contraction and relaxation, disease progression rate may also be different according to the type of mutations, and so is the LGE increment. In this study, more than half of the subjects with preclinical HCM have MYBPC3 mutation (12), which was well-known to cause elderly-onset HCM (19). Unfortunately, genetic information in mutation carriers who experienced disease progression was not reported in this study.

Axelsson Raja et al. also reported the results of followup CMR in the pediatric overt HCM patients. Only two studies were hitherto published regarding the dynamic change of LGE in adult HCM patients over time $(17,18)$. In the current study, the authors elegantly showed the progressive nature of LGE in the pediatric HCM patients (12). Because we do not have any groundbreaking medication to improve the outcome of overt HCM patients, pathophysiology regarding LGE progression should be investigated in depth. This is especially important in pediatric HCM population, in whom the effect of new treatment for blocking progressive $\mathrm{LVH}$ can be maximal. A large-scale registry of CMR in adult HCM patients has already been kicked off (20), and now it is time for a large, prospective CMR registry for pediatric HCM patients to be considered.

\section{Acknowledgements}

None.

\section{Footnote}

Conflicts of Interest: The authors have no conflicts of interest to declare.

\section{References}

1. Maron BJ, Gardin JM, Flack JM, et al. Prevalence of hypertrophic cardiomyopathy in a general population of young adults. Echocardiographic analysis of 4111 subjects in the CARDIA Study. Coronary Artery Risk Development in (Young) Adults. Circulation 1995;92:785-9.

2. Olivotto I, Cecchi F, Poggesi C, et al. Patterns of disease progression in hypertrophic cardiomyopathy: an individualized approach to clinical staging. Circ Heart Fail 2012;5:535-46.

3. Tardiff JC. Assessing the Phenotypic Burden of Preclinical Sarcomeric Hypertrophic Cardiomyopathy-New Assessments to Guide Diagnosis and Management. JAMA Cardiol 2017;2:428-9.

4. Ho CY, Cirino AL, Lakdawala NK, et al. Evolution of hypertrophic cardiomyopathy in sarcomere mutation carriers. Heart 2016;102:1805-12.

5. Alexander PMA, Nugent AW, Daubeney PEF, et al. Long-Term Outcomes of Hypertrophic Cardiomyopathy Diagnosed During Childhood: Results From a National Population-Based Study. Circulation 2018;138:29-36.

6. Choi HM, Yoon YE. Evaluation of Sudden Cardiac Death Risk Using Cardiac Magnetic Resonance. Cardiovascular Imaging Asia 2017;1:67-77.

7. Chan RH, Maron BJ, Olivotto I, et al. Prognostic value of quantitative contrast-enhanced cardiovascular magnetic resonance for the evaluation of sudden death risk in patients with hypertrophic cardiomyopathy. Circulation 2014;130:484-95.

8. Weng Z, Yao J, Chan RH, et al. Prognostic Value of 
LGE-CMR in HCM: A Meta-Analysis. JACC Cardiovasc Imaging 2016;9:1392-402.

9. Elliott PM, Anastasakis A, Borger MA, et al. 2014 ESC Guidelines on diagnosis and management of hypertrophic cardiomyopathy: the Task Force for the Diagnosis and Management of Hypertrophic Cardiomyopathy of the European Society of Cardiology (ESC). Eur Heart J 2014;35:2733-79.

10. O'Mahony C, Jichi F, Pavlou M, et al. A novel clinical risk prediction model for sudden cardiac death in hypertrophic cardiomyopathy (HCM risk-SCD). Eur Heart J 2014;35:2010-20.

11. Mentias A, Raeisi-Giglou P, Smedira NG, et al. Late Gadolinium Enhancement in Patients With Hypertrophic Cardiomyopathy and Preserved Systolic Function. J Am Coll Cardiol 2018;72:857-70.

12. Axelsson Raja A, Farhad H, Valente AM, et al. Prevalence and Progression of Late Gadolinium Enhancement in Children and Adolescents with Hypertrophic Cardiomyopathy. Circulation 2018;138:782-92.

13. El Saiedi S, Behairy NH, Kharabish A, et al. Delayed Myocardial Enhancement in Pediatric Hypertrophic Cardiomyopathy: Correlation with LV Function, Echocardiography, and Demographic Parameters. Pediatr Cardiol 2017;38:1024-31.

14. Chaowu Y, Shihua Z, Jian L, et al. Cardiovascular magnetic resonance characteristics in children with hypertrophic

Cite this article as: Choi HM, Kim HK. Progressive nature of myocardial fibrosis in pediatric hypertrophic cardiomyopathy: from mutation carrier to myocardial fibrosis. Ann Transl Med 2018;6(Suppl 1):S52. doi: 10.21037/atm.2018.10.07 cardiomyopathy. Circ Heart Fail 2013;6:1013-20.

15. Spinner JA, Noel CV, Denfield SW, et al. Association of Late Gadolinium Enhancement and Degree of Left Ventricular Hypertrophy Assessed on Cardiac Magnetic Resonance Imaging With Ventricular Tachycardia in Children With Hypertrophic Cardiomyopathy. Am J Cardiol 2016;117:1342-8.

16. Valente AM, Lakdawala NK, Powell AJ, et al. Comparison of echocardiographic and cardiac magnetic resonance imaging in hypertrophic cardiomyopathy sarcomere mutation carriers without left ventricular hypertrophy. Circ Cardiovasc Genet 2013;6:230-7.

17. Choi HM, Kim KH, Lee JM, et al. Myocardial fibrosis progression on cardiac magnetic resonance in hypertrophic cardiomyopathy. Heart 2015;101:870-6.

18. Todiere G, Aquaro GD, Piaggi P, et al. Progression of myocardial fibrosis assessed with cardiac magnetic resonance in hypertrophic cardiomyopathy. J Am Coll Cardiol 2012;60:922-9.

19. Niimura H, Patton KK, McKenna WJ, et al. Sarcomere protein gene mutations in hypertrophic cardiomyopathy of the elderly. Circulation 2002;105:446-51.

20. Kramer CM, Appelbaum E, Desai MY, et al. Hypertrophic Cardiomyopathy Registry: The rationale and design of an international, observational study of hypertrophic cardiomyopathy. Am Heart J 2015;170:223-30. 